\title{
Development of a Selective Medium for the Fungal Pathogen Fusarium graminearum Using Toxoflavin Produced by the Bacterial Pathogen Burkholderia glumae
}

\author{
Boknam Jung ${ }^{1}$, Sehee Lee ${ }^{1}$, Jiran Ha ${ }^{1}$, Jong-Chul Park ${ }^{2}$, Sung-Sook Han ${ }^{3}$, Ingyu Hwang ${ }^{4}$, Yin-Won Lee ${ }^{4}$ and \\ Jungkwan Lee ${ }^{1 *}$ \\ ${ }^{1}$ Department of Applied Biology, Dong-A University, Busan 604-714, Korea \\ ${ }^{2}$ Department of Rice and Winter Cereal Crop, National Institute of Crop Science, Rural Development Administration, Iksan 570- \\ 080, Korea \\ ${ }^{3}$ Crop Environment Division, National Institute of Crop Science, Rural Development Administration, Suwon 441-857, Korea \\ ${ }^{4}$ Department of Agricultural Biotechnology, Seoul National University, Seoul 151-921, Korea
}

(Received on July 14, 2013; Revised on September 1, 2013; Accepted on September 24, 2013)

\begin{abstract}
The ascomycete fungus Fusarium graminearum is a major causal agent for Fusarium head blight in cereals and produces mycotoxins such as trichothecenes and zearalenone. Isolation of the fungal strains from air or cereals can be hampered by various other airborne fungal pathogens and saprophytic fungi. In this study, we developed a selective medium specific to $F$. graminearum using toxoflavin produced by the bacterial pathogen Burkholderia glumae. F. graminearum was resistant to toxoflavin, while other fungi were sensitive to this toxin. Supplementing toxoflavin into medium enhanced the isolation of $F$. graminearum from rice grains by suppressing the growth of saprophytic fungal species. In addition, a medium with or without toxoflavin exposed to wheat fields for $1 \mathrm{~h}$ had $84 \%$ or $25 \%$, respectively, of colonies identified as $F$. graminearum. This selection medium provides an efficient tool for isolating $F$. graminearum, and can be adopted by research groups working on genetics and disease forecasting.
\end{abstract}

Keywords : Burkholderia, Fusarium, Fusarium head blight, selective medium, toxoflavin

The genus Fusarium consists of more than 100 fungal species, most of which cause severe diseases in various plants. Fusarium head blight (FHB) has threatened the production of major cereal crops including wheat, barley, rice and maize (Desjardins et al., 2000; Lee et al., 2009, 2012; Leslie and Summerell, 2006). In addition to direct yield losses by this disease, causal agents for FHB produce mycotoxins such as trichothecenes and zearalenones that cause feed refusal, vomiting, diarrhea, dermatitis, hemorrhage,

\footnotetext{
*Corresponding author.

Phone) +82-51-200-7554, FAX) +82-51-2007505

E-mail) jungle@dau.ac.kr
}

abortion, and breeding problems in farm animals (Desjardins, 2006; Leslie and Summerell, 2006). Trichothecenes also has been known as a virulence factor on host plants (Cumagun et al., 2005; Proctor et al., 1995). Although several Fusarium species including $F$. avenaceum, F. culmorum, and $F$. poae can cause $\mathrm{FHB}$, the ascomycetous fungus $F$. graminearum (teleomorph: Gibberella zeae) is the most important causal agent for FHB (Leslie and Summerell, 2006).

Isolation of fungal pathogens from either infected plants or air and soil environments is important for many research areas including population genetics, disease diagnosis, and disease forecasting. Many researchers have focused on the development of selective media for specific fungal pathogens. The best known selective medium for Fusarium isolation is peptone-pentachloronitrobenzene (PCNB) medium (Papavizas, 1967). PCNB was formerly registered as a fungicide and used to suppress contamination of zygomycetes. However, the potential of PCNB as a hazardous air pollutant and possible human carcinogen resulted in the cancelation of PCNB registration as an agricultural chemical in many countries. Other selective media based on modified PCNB medium were also used for isolation of Fusarium species.

Previously, we showed that FHB symptoms in rice resemble bacterial rice grain rot caused by Burkholderia glumae, which is the most important bacterial disease of rice in Japan, Korea, and Taiwan (Kim et al., 2004; Lee et al., 2009; Urakami et al., 1994). This bacterial pathogen produces toxoflavin, which is a major virulence factor (Kim et al., 2004). Toxoflavin produces superoxide and hydrogen peroxide during autorecycling oxidation processes under oxygen and light, which damages eukarytotic cells (Kim et al., 2004; Nagamatsu et al., 1982). Discovery of the $t f l A$ gene, encoding the toxoflavin degradation enzyme, led to its use as a selection marker system in plants (Koh et al., 2011). Hence, we hypothesized that $t f l A$ regulated by a 
constitutive fungal promoter could be used as a selection marker for fungal transformation in Fusarium species. However, Fusarium species were not sensitive to toxoflavin, while most other fungal genera including Magnaporthe, Penicillium, Aspergillus, and Collectotrichum were highly sensitive to this toxin. This result suggested that toxoflavin could be used as a selective medium for Fusarium species. This study showed that a medium containing toxoflavin was efficient for isolation of Fusarium species from grains and fields and can be widely used by researchers working on disease diagnosis, population genetics, and disease forecasting.

Toxicity test of toxoflavin against various fungal species. Fungal strains including $F$. graminearum, $F$. oxysporum, Magnaporthe oryzae, Colletotrichum gloeosporioides, and Penicillium sp. were inoculated on yeast malt agar (YMA) (Harris, 2005), potato dextrose agar (PDA), minimal medium (MM), and complete medium (CM) (Leslie and Summerell, 2006) with various concentrations of toxoflavin synthesized as described by Nagamatsu (2001). Toxoflavin was dissolved in distilled water and added into the autoclaved media after cooling down by $50^{\circ} \mathrm{C}$. After inoculating each fungal strain onto the medium, the plates were incubated at $25^{\circ} \mathrm{C}$ under white fluorescent lights, and mycelial growth was measured every $24 \mathrm{~h}$ until 5 days after inoculation. The experiments were repeated three times with three replicates and Tukey test using SPSS 12.0 software (SPSS Inc., Chicago, USA) was performed to examine the significant differences $(\mathrm{P}<0.05)$ of mycelia growth among the mean values of the samples.

At $5 \mathrm{mg}$ toxoflavin/l, the mycelial growth of $M$. oryzae, C. gloeosporioides, and Penicillium sp. was slow compared to that in the absence of toxoflavin, while two Fusarium species, $F$. graminearum and $F$. oxysporum, showed the same growth rate as in the medium without toxoflavin (Fig. 1). As the toxoflavin concentration increased, mycelial growth was increasingly inhibited in all the fungal species except Fusarium species, which had a similar growth rate up to $80 \mathrm{mg}$ toxoflavin/l (Fig. 1). The PDA, YMA, MM, and CM media all showed similar levels of fungal inhibition (data not shown).

To determine whether the $F$. graminearum strains present in nature are resistant to toxoflavin, we isolated $200 \mathrm{~F}$. graminearum strains from rice and wheat in southern provinces and from maize in the eastern province of Korea. Each fungal strain was identified based on morphological characteristics including pigmentation, hyphal growth, and

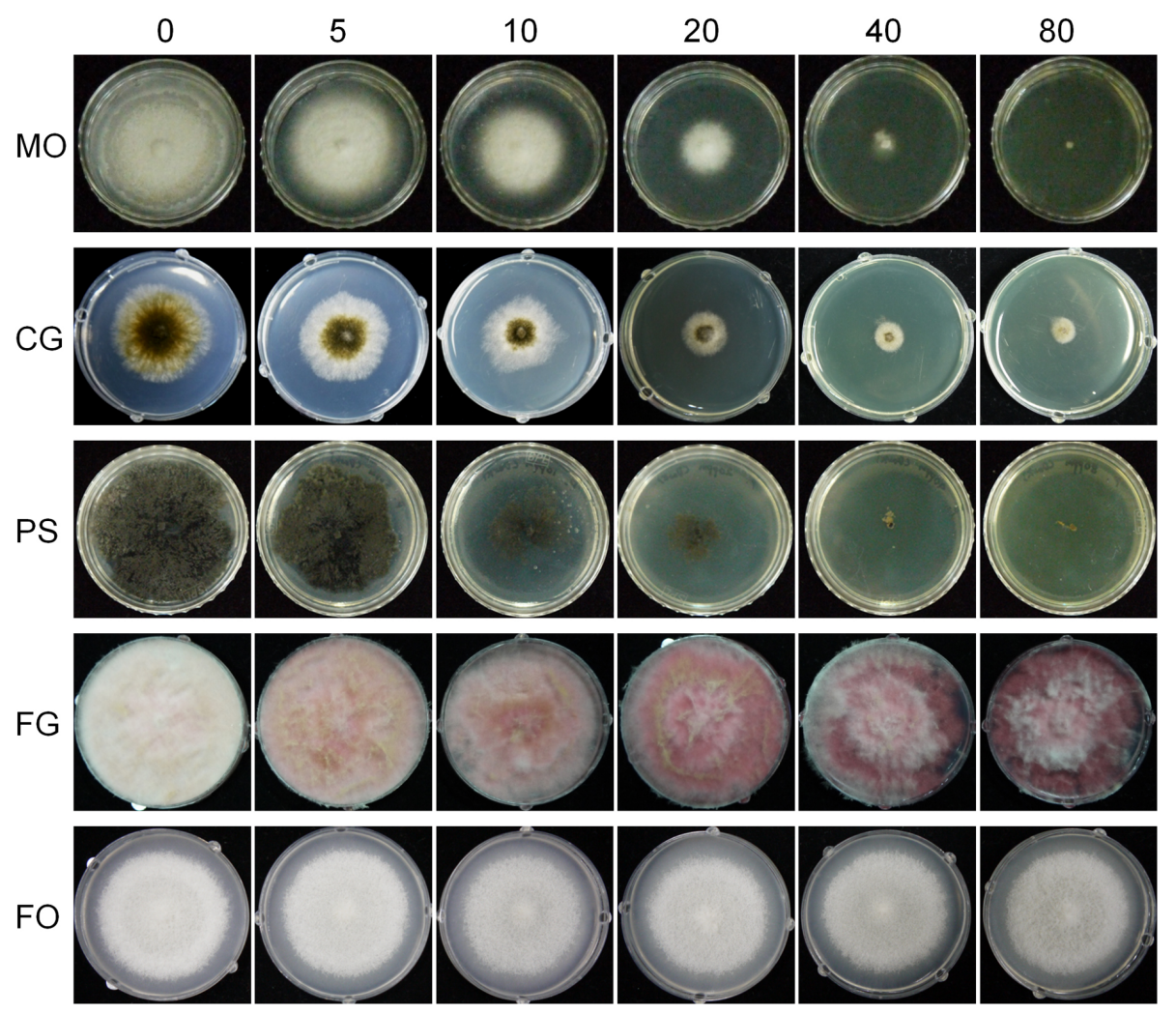

Fig. 1. Toxicity test of toxoflavin against various fungal species. The numbers above the figure indicate the concentration of toxoflavin $\left(\mathrm{mg}^{-1}\right)$ added into minimal medium. MO, Magnaporthe oryzae; CG, Colletotrichum gloeosporioides; PS, Penicillium sp.; FG, Fusarium graminearum; FO, F. oxysporum. Photographs were taken after 5-day incubation at $25^{\circ} \mathrm{C}$. 
spores, and partial translation elongation factor 1-alpha (TEF) DNA sequences. Fungal genomic DNA was extracted from mycelia cultured on CM agar as previously described (Chi et al., 2009). The PCR primers, efl (forward primer; 5'-ATGGGTAAGGA(A/G)GACAAGAC-3') and ef2 (reverse primer; 5'-GGA(G/A)GTACCAGT(G/C)ATCATGTT-3') (O'Donnell et al., 1998), were used to amplify TEF DNA sequences. The primers were synthesized at an oligonucleotide synthesis facility (Cosmotech, Busan, Korea) and diluted to $20 \mu \mathrm{M}$ in sterilized water. PCR was performed as previously described (O'Donnell et al., 1998) and the PCR product was purified using QIAquick PCR purification kit (Qiagen) by following the manufacturer's instruction and directly sequenced in the National Instrumentation Center for Environmental Management (Seoul National University, Seoul, Korea). The sequences were compared to the NCBI database with standard nucleotide BLAST (http://blast.ncbi.nlm.nih.gov/Blast.cgi/). Out of 200 strains, 172 and 28 strains belonged to lineage 6 (F. asiaticum) and lineage 7 ( $F$. graminearum), respectively. When the strains were inoculated on MM containing $80 \mathrm{mg}$ toxoflavin/l, all strains grew as well as in the MM alone, indicating that the $F$. graminearum field population was adaptable to toxoflavin.

Several bacterial pathogens including B. glumae produce toxoflavin, which is a major virulence factor against plant hosts (Kim et al., 2004). The low concentration of toxoflavin $(1-6 \mathrm{mg} / \mathrm{l})$ results in cytotoxicity to various plants including corn, Arabidopsis, tobacco, and cucumber (Koh et al., 2011). Interestingly, detoxification of toxoflavin is triggered by one gene, tflA (Kim et al., 2004). The toxicity to eukaryotic cells and simple detoxification system led to the use of toxoflavin and the $t f l A$ gene as a positive selection system for plant transformation (Koh et al., 2011), and as a possible selection marker for Fusarium transformation in this study. However, all F. graminearum strains tested grew well even with $>100 \mathrm{mg}$ toxoflavin/l, while other fungal species such as M. oryzae, Penicillium sp., and $C$. gloeosporioides were more sensitive to this toxin. This result suggested that toxoflavin and the $t f l A$ gene are not suitable selection markers of Fusarium species, although toxoflavin could be developed as a selective medium for Fusarium species.

Isolation of fungal strains from rice seeds. The toxicity test results suggested that Fusarium species could be selectively isolated from grains. Surface sterilized rice grains were placed on PDA with or without $80 \mathrm{mg}$ toxoflavin/l. In brief, fungal strains were isolated from rice seeds, including the husks, collected from one southern province (Gyeongnam) of Korea during Setember 2012. For isolating fungal strains from rice seeds, 200 seeds were soaked in $1 \%(\mathrm{w} / \mathrm{v})$ sodium hypochlorite for $2 \mathrm{~min}$, and then rinsed in sterile water for 2 min. After surface sterilization, 100 seeds were placed on PDA amended with $50 \mathrm{mg}$ kanamycin/l, and 100 seeds were also placed on PDA containing both $50 \mathrm{mg}$ kanamycin and $80 \mathrm{mg}$ toxoflavin/l. The plates were then incubated at $25^{\circ} \mathrm{C}$ for $4-7$ days. Fungal isolates were transferred to fresh PDA, and all cultures were purified by single spore isolation.

Other fungal strains including $F$. oxysporum, C. gloeosporioides, and M. oryzae were provided by the Center for Fungal Genetic Resources (Seoul National University, Seoul, Korea), and F. graminearum GZ03639 lineage 7 strain (Bowden and Leslie, 1999) was used as a reference strain. All fungal strains were maintained on PDA and CM, and stored as conidia suspensions in $20 \%$ (v/v) glycerol at $-80^{\circ} \mathrm{C}$.

Diverse saprophytic fungal genera including Aspergillus and Penicillium were identified on PDA without toxoflavin (Fig. 2), but we did not purify F. graminearum. In contrast, the growth of saprophytic fungi was efficiently inhibited on the PDA containing $80 \mathrm{mg}$ toxoflavin/l (Fig. 2). In total, four fungal strains from 100 rice seeds were grown well and they all were identified as $F$. graminearum based on the morphological characteristics and TEF sequences.

Isolation of fungal strains from air. MM medium containing 0, 20, and $80 \mathrm{mg}$ toxoflavin/l were added to $90 \mathrm{~mm}$ Petri dishes and the plates were exposed to a field of wheat at early anthesis stage in the Dong-A University Research Farm (Gyeongnam province, Korea) in May 2012. Three plates with each concentration of toxoflavin were left open for $1 \mathrm{~h}$ at three different locations $\sim 100 \mathrm{~m}$ apart from each other in the wheat field. The plates were incubated at $25^{\circ} \mathrm{C}$ and the fungal colonies were subcultured onto fresh PDA. Each strain was purified through single spore isolation.

Plates with MM containing 0, 20, and $80 \mathrm{mg}$ toxoflavin/1 were exposed in a wheat field for $1 \mathrm{~h}$. The plates were incubated at $25^{\circ} \mathrm{C}$ for $72 \mathrm{~h}$ and fungal colonies greater than

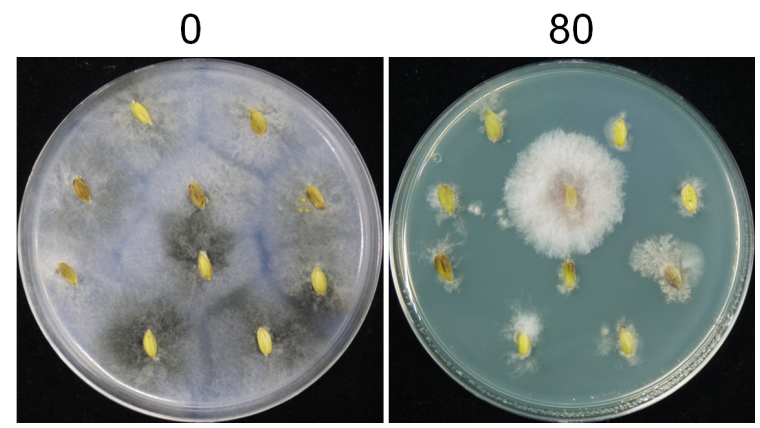

Fig. 2. Isolation of fungal strains from rice grains using a toxoflavin-containing medium. Surface sterilized rice grains were placed on MM containing 0 or $80 \mathrm{mg}$ toxoflavin $\mathrm{l}^{-1}$. Photographs were taken after 4-day incubation at $25^{\circ} \mathrm{C}$. 
0
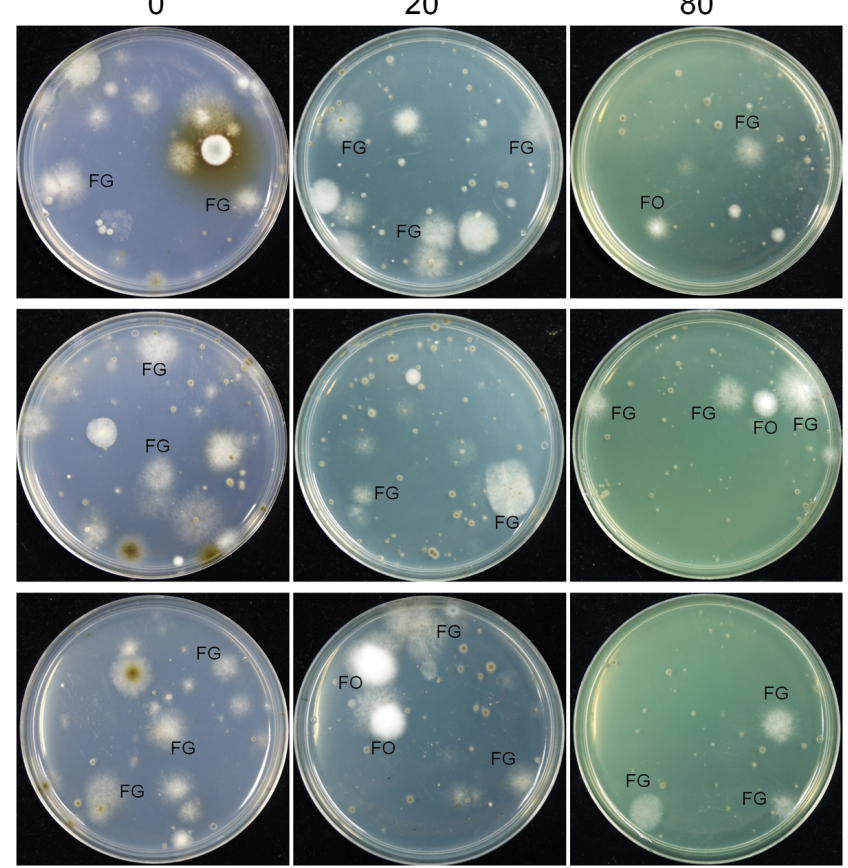

Fig. 3. Isolation of fungal strains from air using a toxoflavincontaining medium. Minimal media containing 0,20 , and $80 \mathrm{mg}$ toxoflavin/L were exposed to a wheat field for $1 \mathrm{~h}$ and the plates were incubated for 3 days at $25^{\circ} \mathrm{C}$. The three rows indicate separate sites within a field (separated by $\sim 100 \mathrm{~m}$ ), with the three replicate plates in each row.

$5 \mathrm{~mm}$ diameter were counted. The number of fungal strains and the variety of fungal species isolated from the media varied significantly with different toxoflavin concentrations (Fig. 3). On MM without toxoflavin, 11.8 fungal strains per plate were isolated, but 6.2 and 3.2 strains per plate were isolated on MM containing 20 and $80 \mathrm{mg}$ toxoflavin/l, respectively. Approximately three Fusarium strains per plate were isolated from all plates regardless of the toxoflavin concentration, while efficiently eliminating other saprophytic fungal species by adding toxoflavin to the medium. Consequently, $25 \%, 42 \%$, and $88 \%$ of strains isolated from media containing 0,20 , and $80 \mathrm{mg}$ toxoflavin/l, respectively, were Fusarium species ( $\sim 95 \%$ and $\sim 5 \%$ were $F$. graminearum lineage 6 and $F$. oxysporum, respectively).

Isolation of specific fungal species from contaminated plants and environment such as air, soil, and water is of significant interest for fungal plant pathologists. It is the first step in many research areas including disease diagnosis, disease forecasting, taxonomy, and population genetics. Specifically, many researchers have focused on the development of selective media for Fusarium species because this genus is one of the most important plant pathogens and exists in various environmental conditions. The most common media for isolation of Fusarium species are based on PCNB (Leslie and Summerell, 2006). PCNB had been registered as a fungicide that inhibits most fungi including zygomycete fungi but allows slow growth of Fusarium, resulting in the prevalent use of this medium for Fusarium isolation. However, most Fusarium species do not form distinctive colonies on PCNB media, and hence all colonies formed on the media must be subcultured (Leslie and Summerell, 2006). In addition, PCNB is a potential carcinogen and its registration was canceled, which led us to develop an alternative to PCNB-based media.

The toxoflavin-based selective medium developed in this study showed a high selectivity for Fusarium species. More than $80 \%$ of fungal strains isolated from both grains and air were Fusarium species, particularly F. graminearum, which may be more prevalent in wheat grains and wheat fields where $F$. graminearum is a major causal pathogen. However, F. oxysporum and F. verticillioides also showed high resistance to toxoflavin, indicating that this medium could also be useful for the isolation of these species in other crops and fields.

The resistance mechanism of Fusarium species against toxoflavin has not yet been elucidated, but may have occurred throughout the long evolutionary relationship between Fusarium and Burkholderia. FHB in rice has not been studied much because it is not considered too damaging for rice, although it has been quite destructive in wheat and barley. Previously, we showed that the FHB symptoms in rice caused by $F$. graminearum resemble B. glumae bacterial rice grain rot, which is one of the most important rice diseases in East Asia (Lee et al., 2009). In that study, rice grains were highly contaminated by $F$. graminearum, and $F$. graminearum strains isolated from other crops such as maize and wheat also triggered severe FHB in rice, indicating that FHB in rice has been as severe as in wheat and barley grown in Korea. Therefore, it is possible for $F$. graminearum to occupy the same niche as B. glumae, and there could be a specific interaction between the two kingdoms. Our future research will focus on elucidating the resistance mechanism of $F$. graminearum against toxoflavin, produced by $B$. glumae, and the interaction between the two pathogens in rice.

\section{Acknowledgements}

This work was supported by Basic Science Research Program through the National Research Foundation of Korea (NRF) funded by the Ministry of Science, ICT and Future Planning (2012003883), a grant provided by Rural Development Administration (PJ0082982013), and a NRF grant funded by the Korean Government (MSIP) (No. 20080061897). We thank Dr. Tomohisa Nagamatsu for providing toxoflavin. 


\section{References}

Bowden, R. L. and Leslie, J. F. 1999. Sexual recombination in Gibberella zeae. Phytopathology 89:182-188.

Chi, M. H., Park, S. Y., Kim, S. and Lee, Y. H. 2009. A quick and safe method for fungal DNA extraction. Plant Pathol. J. 25:108-111.

Cumagun, C. J. R., Bowden, R. L., Jurgenson, J. E., Leslie, J. F. and Miedaner, T. 2004. Genetic mapping of pathogenicity and aggressiveness of Gibberella zeae (Fusarium graminearum) towards wheat. Phytopathology 94:520-526.

Desjardins, A. E. 2006. Fusarium mycotoxins: Chemistry, Genetics and Biology. APS Press, St. Paul, MN, USA. 260 pp.

Desjardins, A. E., Manhanadhar, H. K., Plattner, R. D., Manandhar, G. G., Poling, S. M. and Maragos, C. M. 2000. Fusarium species from Nepalese rice and production of mycotoxins and gibberellic acid by selected species. Appl. Environ. Microbiol. 66:1020-1025.

Harris, S. D. 2005. Morphogenesis in germinating Fusarium graminearum macroconidia. Mycologia 97:880-887.

Kim, J., Kim, J. G., Kang, Y., Jang, J. Y., Jog, G. J., Lim, J. Y., Kim, S., Suga, H., Nagamatsu, T. and Hwang, I. 2004. Quorum sensing and the LysR-type transcriptional activator ToxR regulate toxoflavin biosynthesis and transport in Burkholderia glumae. Mol. Microbiol. 54:921-934.

Koh, S., Kim, H., Kim, J., Goo, E., Kim, Y. J., Choi, O., Jwa, N. S., Ma, J., Nagamatsu, T., Moon, J. S. and Hwang, I. 2011. A novel light-dependent selection marker system in plants. Plant Biotechnol. J. 9:348-358.

Lee, J., Chang, I., Kim, H., Yun, S. H., Leslie, J. F. and Lee, Y. W. 2009. Genetic diversity and fitness of Fusarium graminearum populations from rice in Korea. Appl. Environ. Microbiol. 75:3289-3295.
Lee, J., Kim, H., Jeon, J.-J., Kim, H.-S., Zeller, K. A., Carter, L. L. A., Leslie, J. F. and Lee, Y.-W. 2012. Population structure of and mycotoxin production by Fusarium graminearum from maize in South Korea. Appl. Environ. Microbiol. 78:21612167.

Leslie, J. F. and Summerell, B. A. 2006. The Fusarium laboratory manual. Blackwell Professional, Ames, IA, USA.

Nagamatsu, T. 2001. Synthesis, transformation, and biological activities of 7-azaperidine antibiotics: Toxoflavin, fervenulin, reumycin and their analogs. Recent Res. Devel. Org. Bioorg. Chem. 4:97-121.

Nagamatsu, T., Hashiguchi, Y., Sakuma, Y. and Yoneda, F. 1982. Autorecycling oxidation of amines to carbonyl compound catalized by 3, 4-disubstituted 4-deazatoxoflavin derivatives. Chem. Lett. 11:1309-1312.

O’Donnell, K., Kistler, H. C., Cigelnik, E. and Ploetz, R. C. 1998. Multiple evolutionary origins of the fungus causing Panama disease of banana: Concordant evidence from nuclear and mitochondrial gene genealogies. Proc. Natl. Acad. Sci. USA 95:2044-2049.

Papavizas, G. C. 1967. Evaluation of various media and antimicrobial agents for isolation of Fusarium from soil. Phytopathology 57:848-852.

Proctor, R. H., Hohn, T. M. and McCormick, S. P. 1995. Reduced virulence of Gibberella zeae caused by disruption of a trichothecene toxin biosynthetic gene. Mol. Plant-Microbe Interact. 8:593-601.

Urakami, T., Ito-Yoshida, C., Araki, H., Kijima, T., Suzuki, K. and Komagata, K. 1994. Transfer of Pseudomonas plantarii and Pseudomonas glumae to Burkholderia as Burkholderia spp. and description of Burkholderia vandii sp. Nov. Int. J. Syst. Bacteriol. 44:235-245. 